\title{
Training Emergency Physicians in Ultrasound- guided Fascia Iliaca Compartment Blocks: Lessons in Change Management
}

Juliana Wilson ${ }^{1}$, Kaylah Maloney ${ }^{2}$, Kelly Bookman ${ }^{3}$, Jason W. Stoneback ${ }^{4}$, Vaughn A. Browne ${ }^{3}$, Adit Ginde $^{3}$, Mary Wallace ${ }^{5}$, Gabrielle Jacknin ${ }^{6}$, Ethan Cumbler ${ }^{7}$, Resa E. Lewiss ${ }^{2}$

1. Emergency Medicine, University of Colorado, Denver, USA 2. Emergency Medicine, Thomas Jefferson University, Philadelphia, USA 3. Emergency Medicine, University of Colorado, Aurora, USA 4. Orthopedics, University of Colorado, Aurora, USA 5. Internal Medine, University of Colorado, Aurora, USA 6. Pharmacy, University of Colorado Hospital, Denver, USA 7. Internal Medicine, University of Colorado, Aurora, USA

Corresponding author: Juliana Wilson, juliana.wilson@ucdenver.edu

\section{Abstract}

\section{Study objectives}

Older adults who sustain hip fractures are susceptible to high rates of morbidity and mortality. The systemic administration of opioids is associated with side effects disproportionately affecting the elderly. The ultrasound-guided fascia iliaca compartment block procedure (FICB) is associated with a reduced patient need for oral and parenteral opioids and with improved functional outcomes. We designed a multidisciplinary quality improvement initiative to train emergency physicians (EPs) to perform the ultrasoundguided FICB procedure for geriatric hip fracture patients. We examined the lessons derived from the EPs' resistance to implementing a practice-changing behavior.

\section{Methods}

This study was a prospective observational cohort study. We included all emergency department (ED) patients > 65 years with X-ray confirmation of isolated hip fractures. We also enrolled the treating EPs. Patients were enrolled from March 2016 to January 2017 in an urban, academic ED with 100,000 annual visits. The ED ultrasound faculty trained ED faculty and residents in the FICB procedure. Seventeen of 50 attending EPs completed the training: classroom lecture and online narrated video instruction. The handson sessions consisted of three stations: scan a human model volunteer to review the sonoanatomy, practice the needle technique using a Blue Phantom ${ }^{\mathrm{TM}}$ Regional Anesthesia Ultrasound Training Block Model (Simulaids, Inc., NY, US), and practice the needle technique using a static simulator. We created a multidisciplinary geriatric hip fracture order set for the electronic medical record. The attending EPs, caring for eligible patients, were asked to complete a Research Electronic Data Capture (REDCap) survey, and we analyzed the data using descriptive statistics.

Received 10/10/2018

Review began 04/17/2019 Review ended 05/22/2019 Published 05/28/2019

() Copyright 2019 Wilson et al. This is an open access article distributed under the terms of the Creative Commons Attribution License CC-BY 3.0., which permits unrestricted use, distribution, and reproduction in any medium, provided the original author and source are credited.

\section{Results}

We enrolled 77 geriatric hip fracture patients. Two of the 77 patients received FICB. Thirty-two EPs participated as providers for these patients while $97 \%$ of these providers completed the post-intervention survey. Providers used the geriatric hip fracture order set in 10 of 77 encounters. Most EPs did not perform the block because they were not trained or did not feel comfortable performing it.

\section{Conclusion}

Despite the efficacy supported by the literature and training sessions offered, the EPs in this study did not adopt the FICB procedure. Future efforts could include developing a FICB on-call team, increasing the proportion of trained EPs through initial supervised hands-on practice, and partnering financial or education incentives with getting trained.

Categories: Emergency Medicine, Pain Management

Keywords: regional anesthesia, point of care ultrasound, change management, emergency medicine

\section{Introduction}

Older adults who sustain hip fractures are susceptible to high rates of morbidity and mortality [1]. Pain control for this population is difficult because opioids, the most commonly used medications, are associated with safety concerns, such as respiratory depression, altered mental status, and an increased incidence of falls. Regional anesthesia performed under direct ultrasound visualization provides excellent anesthesia, a reduced need for oral and parenteral opioids, and improved functional outcomes [2-3]. We designed a multi-disciplinary quality improvement initiative to train emergency physicians (EPs) in ultrasound-guided fascia iliaca compartment blocks (FICB). The original intent of the study was to compare demographics, 


\section{Cureus}

length of stay, total opiate and non-opiate pain medications used, as well as outcomes between geriatric patients that received the FICB and those that did not. As we implemented the training and study and began data collection, we found that few FICBs were being performed. In response to this finding, the project pivoted to understanding barriers to the adoption of this new skill. We examined the lessons in change management derived from the EPs resistance as well as barriers to implementing a quality improvement program and changing the EPs' behavior.

\section{Materials And Methods}

We studied all emergency department (ED) patients greater than 65 years of age, with radiographic confirmation of isolated hip fracture and who were able to give written informed consent. Patients were enrolled from March 2016 to January 2017 in an urban, academic ED with 100,000 annual visits. We also enrolled the treating EPs. The ED ultrasound faculty organized voluntary training sessions following faculty department meetings that consisted of human model scanning, Blue Phantom ${ }^{\mathrm{TM}}$ Regional Anesthesia Ultrasound Training Block station (Simulaids, Inc., NY, US), and a muscle nerve fascia static simulator [4]. There were flipped classroom online reading assignments, an online narrated training video, and a portable document format (.pdf) document available to all EPs. The images used in the training sessions are given in the Appendix.

Seventeen of 50 attending EPs completed the training. A geriatric hip fracture order set was created in the electronic medical record. This standardized the equipment, medications, and tests ordered for the procedure. This also minimized EPs missing certain orders prior to the nerve block. To simplify the process, we pre-filled individual plastic bags, containing all items needed to perform the procedure. For each geriatric hip fracture patient, we collected demographic information, hip fracture characteristics, pain medication administered, and hospital length of stay. We collected prospective data and performed a descriptive analysis of the ED attending physicians, the procedure performance, and the order set utilization. The attending EPs, caring for eligible patients, were asked to complete a Research Electronic Data Capture (REDCap) survey. This REDCap survey assessed which EPs performed the block, those who did not, the reasoning for not performing, and whether or not they used the order set. We included the use of the order set as it was designed to decrease barriers to the implementation of the FICB by bundling orders for medications, neurologic checks, and monitoring. For this study, R, version 3.4.0, was used for all statistical analysis. The mean and standard deviation were reported for continuous data and percentages were reported for categorical data.

\section{Results}

There were 77 geriatric patients with hip fractures that met inclusion criteria for enrollment (Table 1).

\begin{tabular}{|c|c|c|c|}
\hline Variable & All Patients $(\mathrm{n}=77,100 \%)$ & Block Patients (n = 2, 2.60\%) & No Block Patients ( $n=75,97.40 \%)$ \\
\hline Age (years) (mean $\pm s d)$ & $81.32 \pm 9.28$ & $79.5 \pm 10.61$ & $81.37 \pm 9.32$ \\
\hline Older than $90(\%)$ & 22.08 & 0 & 22.67 \\
\hline \multicolumn{4}{|l|}{$\operatorname{Sex}(\%)$} \\
\hline Male & 29.87 & 50.00 & 29.33 \\
\hline Female & 70.13 & 50.00 & 70.67 \\
\hline \multicolumn{4}{|l|}{ Race (\%) } \\
\hline Caucasian & 79.22 & 100 & 78.67 \\
\hline African American & 12.99 & 0 & 13.33 \\
\hline Asian & 2.60 & 0 & 2.67 \\
\hline Other & 5.19 & 0 & 5.33 \\
\hline \multicolumn{4}{|l|}{ Hispanic (\%) } \\
\hline Yes & 9.09 & 0 & 9.33 \\
\hline No & 90.91 & 100 & 90.6 \\
\hline
\end{tabular}

TABLE 1: Shows the demographic data for the 77 patients enrolled during the study period 


\section{Cureus}

Thirty-two treating EPs participated in the study. The EPs completed a survey for each geriatric hip fracture patient that they treated. We had a greater than $97 \%$ response rate to the post-intervention survey. Of the 77 patients, two received FICB (Figure 1).

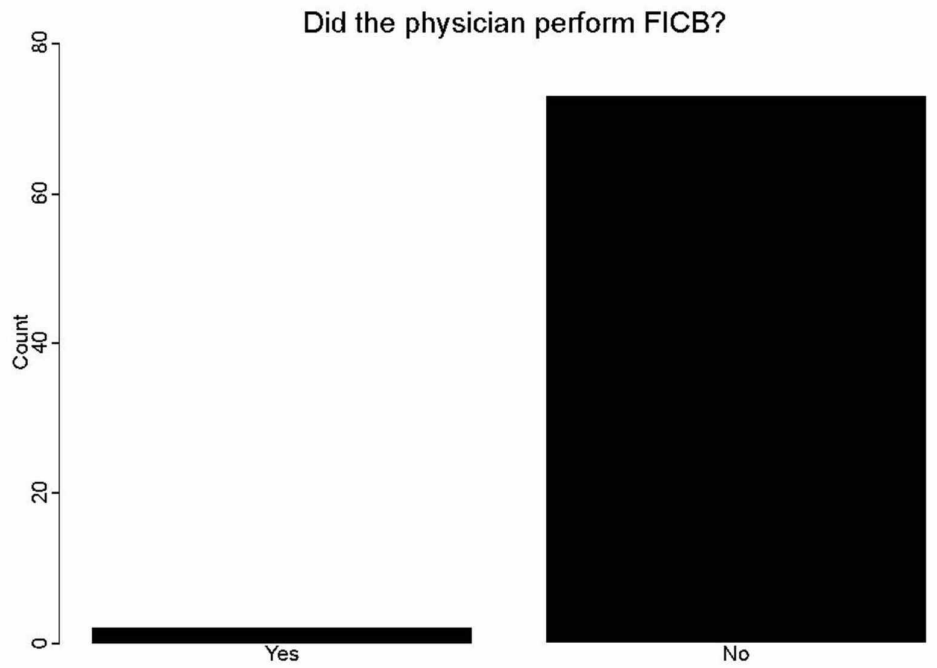

FIGURE 1: Ratio of physicians who performed the fascia iliaca compartment block (FICB)

The more common reasons that physicians did not perform FICB: not trained (24 of 77: 31\%) and not comfortable performing FICB, despite completing the training (13 of 77: 17 \%) (Figure 2).

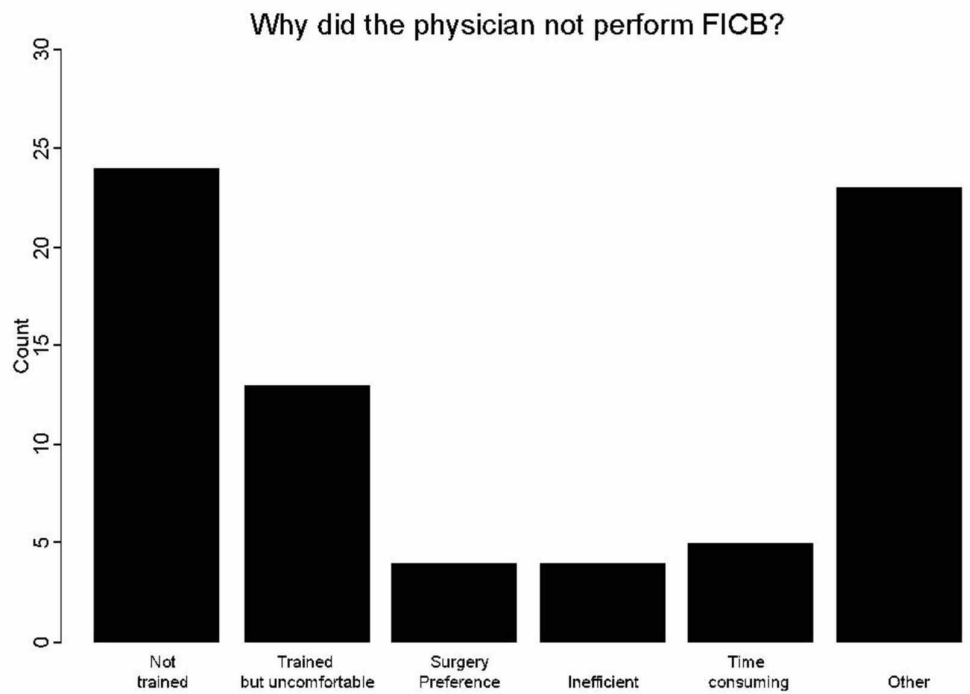

FIGURE 2: Reasons the EP did not perform the fascia iliaca compartment block (FICB)

A few of the physicians (10 of 77: 12.99\% ) used the ED geriatric hip fracture order set (Figure 3). 


\section{Cureus}

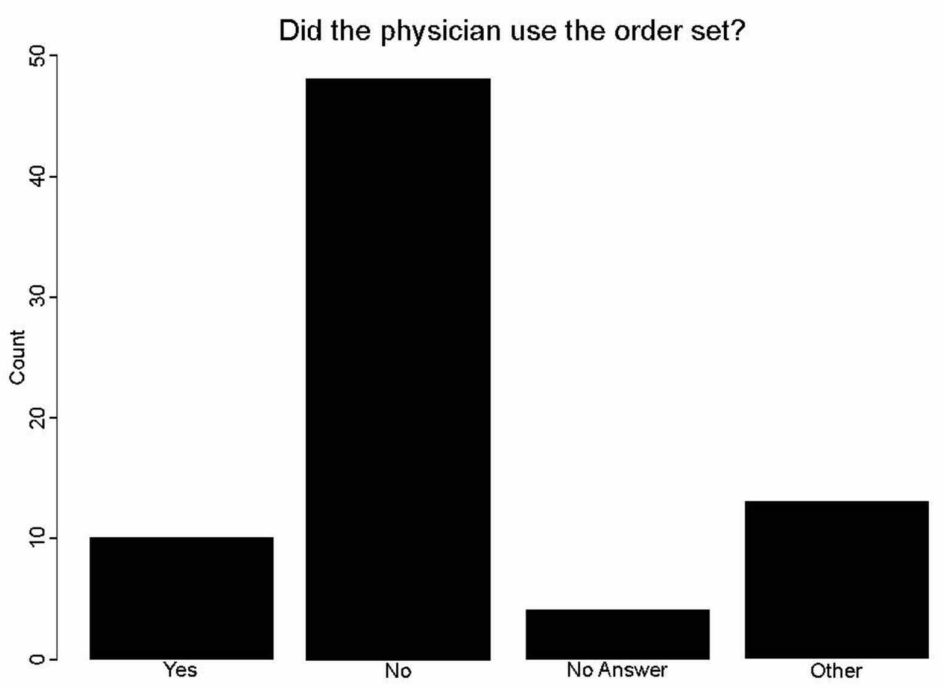

FIGURE 3: Ratio of physicians who used the geriatric hip fracture order set

The two patients who received FICB had a shorter total length of stay overall in the hospital as compared to the no block patients (82.38 and 143.54 hours, respectively) (Table 2). 


\section{Cureus}

\section{Variable}

Height ( meters) (mean $\pm \mathrm{sd})$

Weight $(\mathrm{kg})($ mean $\pm \mathrm{sd})$

Body Mass Index (mean $\pm \mathrm{sd}$ )

Length of Stay-ED (hours) (mean \pm sd)

Pre-Operation Time (hours) (mean \pm sd)

Length of Stay-Total (hours) (mean \pm sd)

Admitting Service (\%)

Acute Care Elderly

Acute Care Surgery-Trauma

General Surgery

Hospitalist-Hospital Medicine Service

Medicine 2

Medicine 3

Orthopedic Surgery

Orthopedics

Vascular Surgery

Unknown

Hip Fracture Diagnosis (\%)

Discharge Disposition (\%)

Discharge to another facility

Home/Hospice

Rehab Facility

Skilled Nursing Facility

Order Set Performed (\%)

Fracture Type (\%)

Femoral neck

Intertrochanteric Fracture

Intracapsular Fracture

Subtrochanteric Fracture

Unknown
All Patients $(n=77,100 \% \quad$ Block Patients $(n=2$, )

$1.66 \pm 0.12$

$63.51 \pm 15.21$

$23.12 \pm 4.81$

$5.37 \pm 5.27$

$25.41 \pm 14.4$

$141.93 \pm 140.75$

1.30

7.79

1.30

1.30

1.30

1.30

54.55

23.38

1.30

6.49

96.10

1.30

24.68

6.49

67.53

19.48

35.14

50.00

1.35

4.05

9.46
$2.60 \%$ )

$1.73 \pm 0.07$

$63.25 \pm 17.61$

$20.95 \pm 4.16$

$5.05 \pm 0.04$

$24.82 \pm 1.59$

$82.28 \pm 18.6$
$143.54 \pm 142.3$

No Block Patients $(\mathrm{n}=\mathbf{7 5}$,

97.40\%)

$1.65 \pm 0.12$

$63.52 \pm 15.28$

$23.18 \pm 4.83$

$5.38 \pm 5.34$

$25.43 \pm 14.62$
1.33

8.00

1.33

1.33

1.33

1.33

53.33

24.00

1.33

6.67

96.00

1.33

25.33

5.33

68.00

17.33

100

36.11

48.61

1.39

4.17

9.72

TABLE 2: Demographic information, admitting service and disposition of geriatric hip fracture patients

\section{Discussion}

This quality improvement study can be viewed as a lesson in change management. Although there have 


\section{Cureus}

been many studies conducted to explore aspects of practice change, implementing change in healthcare remains challenging [5-6]. Part of change management is forming a powerful guiding coalition and creating and communicating a vision [7-9]. Change management and the implementation of a new practice also involves several steps. These steps include: (1) knowledge, which includes the initial step of educating and communicating to staff; (2) persuasion or using incentives to encourage staff involvement; (3) decision or the staff making the choice to accept change; (4) implementation of the new practice; and (5) confirmation in which the staff acknowledges the benefits of the practice change [10]. There were several steps that presented as roadblocks in the implementation of the FICB in this study. The initial step in education was accomplished by the multimodal training implemented. However, some EPs stated that they were uncomfortable performing the FICB independently despite attending training. The second step of persuasion or incentivizing staff also posed an obstacle as translating the enthusiasm of the early adopting EPs to influence more of the majority was a critical challenge in this initiative. Without the mindset that the FICB would be a great change within the department, the adoption of this skill was an obstacle. In future implementations, there are at least two ways in which department leadership could encourage more EP involvement: incentivizing or mandating. With the obstacles presented in the first two steps of the implementation, we were unable to proceed to further steps. The limitations of this study include the single urban academic center setting. The clinical environment is very time-efficient so arguably more FICBs would be performed if patients were in the ED longer. A second limitation was our difficulty in motivating faculty to attend the training. Perhaps if mandated or incentivized, more EPs would have been trained.

\section{Conclusions}

We sought to study the impact of implementing a FICB treatment program for geriatric hip fracture patients. We found that we did not anticipate the barriers to the change management of the EP practice. In the future, anticipating these challenges while implementing a quality improvement initiative should aid in its success.

\section{Appendices}

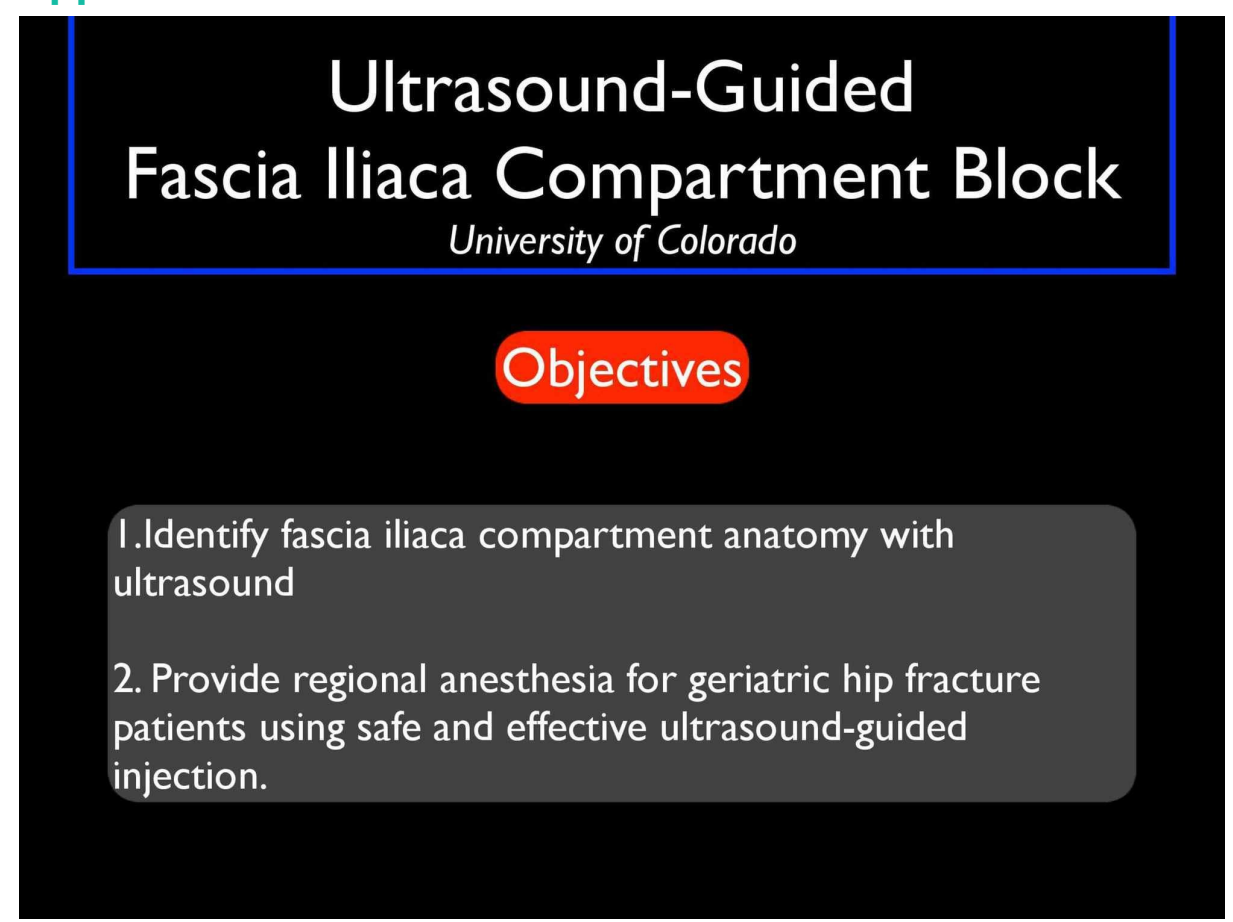

FIGURE 4: Image 1/12 of training presentation 


\section{Cureus}

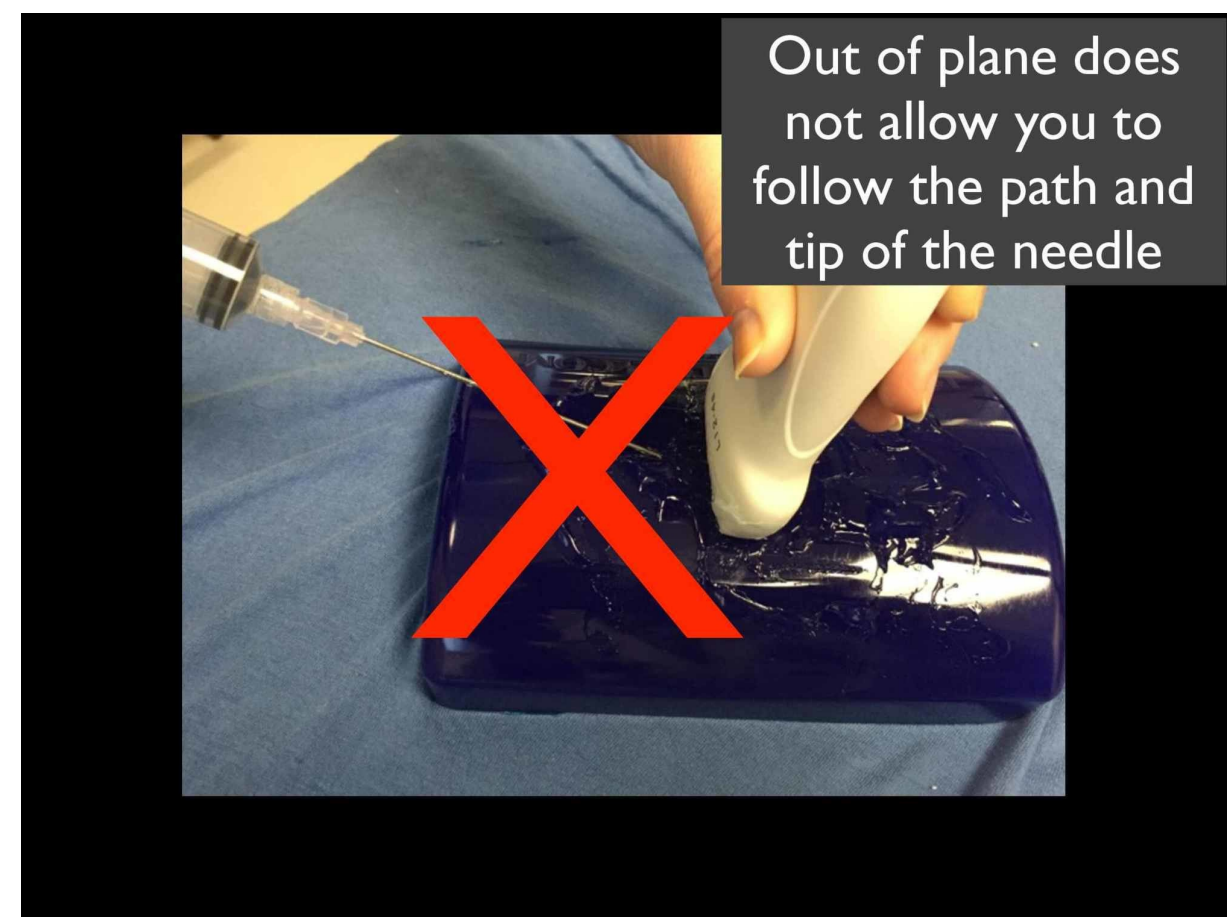

FIGURE 5: Image 2/12 of training presentation

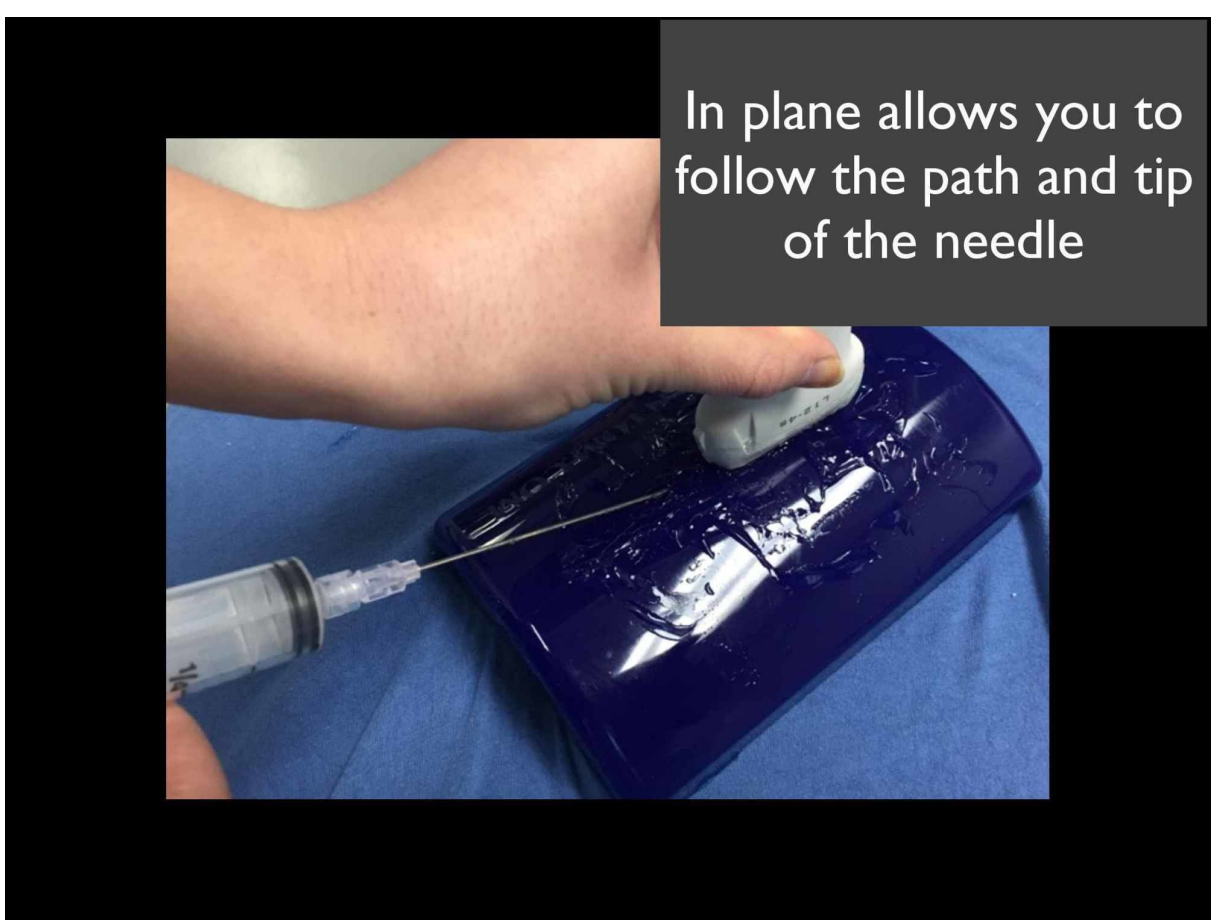

FIGURE 6: Image 3/12 of training presentation 


\section{Cureus}

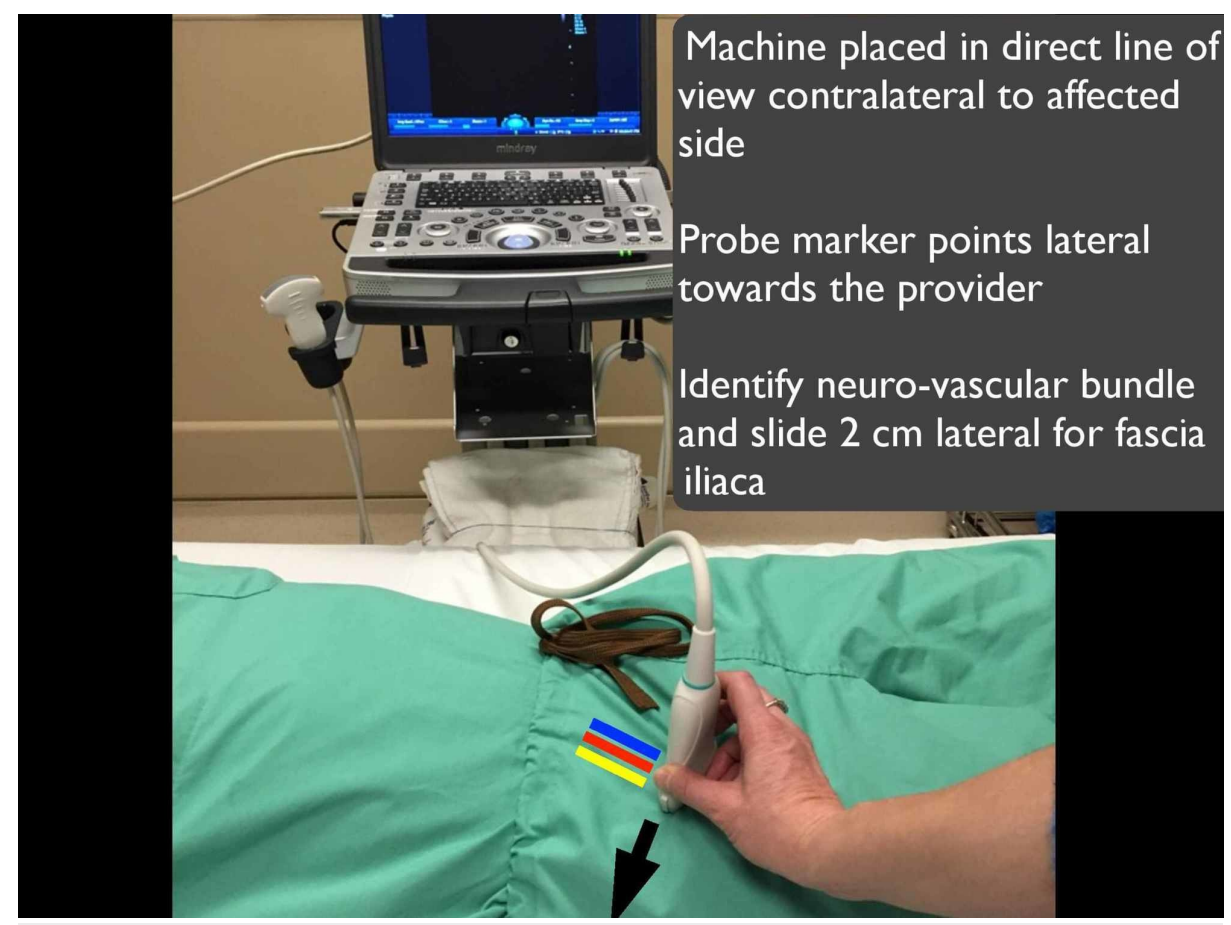

FIGURE 7: Image 4/12 of training presentation

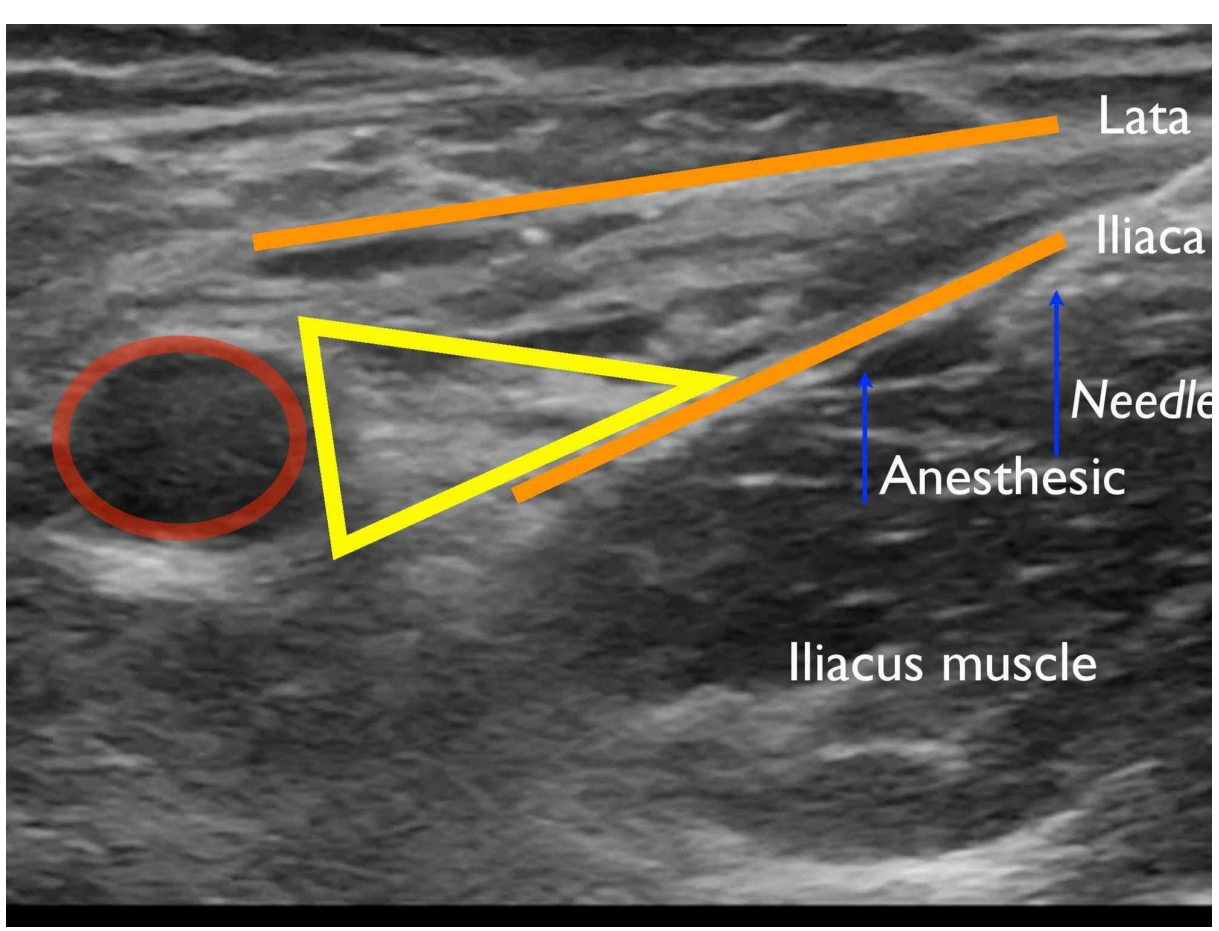

FIGURE 8: Image 5/12 of training presentation 


\section{Cureus}

\section{Medications}

Ropivacaine $0.5 \% 20 \mathrm{ml}$ I vial Lidocaine I\% without epic $(5 \mathrm{ml})$ Normal saline mixing bag $(25 \mathrm{ml})$ Intralipid in Pyxis treatment protocol in book

\section{Equipment}

$5 \mathrm{ml}$ Syringe

$27 \mathrm{~g}$ Needle

$20 \mathrm{ml}$ Syringe (Two)

Blunt tip needle (Two)

20 gauge spinal needle 3.5 inch

FIGURE 9: Image 6/12 of training presentation

\section{Local Anesthetic Systemic Toxicity (LAST)}

Symptoms and signs:

Hypotension

Cardiac: Frequent PVCs, VTach, asystole

Neurologic: tinnitus, metallic taste, confusion, tremor, seizure

Treatment

Any signs or symptoms of LAST, administer lipid emulsion therapy to create a "lipid sink" that draws anesthetic out of cardiac tissue

FIGURE 10: Image 7/12 of training presentation 


\section{Cureus}

\section{Physicians}

- Geriatric Hip Fracture Order Set in Epic

- Informed consent for procedure (not for study)

- Mark site and perform a timeout with ED RN

- Review Indications, Contraindications, Alternatives

- Continuous Pulse Oximetry and Telemetry

- Review medication dose recommendations

- Review symptoms and treatment plan for LAST

- Verify protocol and location and LAST first dose

FIGURE 11: Image 8/12 of training presentation

\section{Contra-Indications}

- No Consent*

- Allergy to amide local anesthetics

(Lidocaine,Bupivacaine, Ropivacaine)

- Infection at block site

- Open fracture or Anatomy which is unsafe

- Unstable patient or New neurologic deficit in affected leg

- Relative:Anticoagulant or antiplatelet medications

- Relative:Any patient with known bleeding disorder

- Relative: Femoral lines (arterial or venous) and recent femoral procedures (angio or bypass)

FIGURE 12: Image 9/12 of training presentation 


\section{Cureus}

\section{Nursing and Techs}

- Ensures patient has working IV

- Administers pain management and records pain per Hip Fracture Order Set

- Assists with obtaining nerve block supplies

- Connects patient to monitor

- Maintains close observation and monitoring during and after procedure for signs of LAST for 30 minutes post procedure

FIGURE 13: Image 10/12 of training presentation

\section{Procedure}

- Maintain constant visualization of needle tip at all times

- Confirm anticipated deposition of anesthetic q $5 \mathrm{~mL}$

- Aspirate q $5 \mathrm{~mL}$ to verify absence of intravascular injection

- Confirm absence of metallic taste, circumoral paresthesia, change in expected mental status q $5 \mathrm{~mL}$

- Monitor for brady/tachy dysrhythmia, dramatic change in blood pressure, seizures

- Record video clips of anatomy pre-injection and serial videos throughout procedure 


\section{Cureus}

\section{Post-Procedure}

- Record absence of LAST signs and symptoms

- Monitor for brady/tachy dysrhythmia, dramatic change in blood pressure, or seizure activity

- Record vital signs q 15 min $\times 2$

- Document volume and dose of all medications used

- Document procedure note

FIGRE 15: Image 12/12 of training presentation

\section{Additional Information}

\section{Disclosures}

Human subjects: Consent was obtained by all participants in this study. University of Colorado issued approval 1402432. Animal subjects: All authors have confirmed that this study did not involve animal subjects or tissue. Conflicts of interest: In compliance with the ICMJE uniform disclosure form, all authors declare the following: Payment/services info: All authors have declared that no financial support was received from any organization for the submitted work. Financial relationships: All authors have declared that they have no financial relationships at present or within the previous three years with any organizations that might have an interest in the submitted work. Other relationships: All authors have declared that there are no other relationships or activities that could appear to have influenced the submitted work.

\section{Acknowledgements}

Supported by NIH/NCATS Colorado CTSI Grant Number UL1 TR001082. Contents are the authors' sole responsibility and do not necessarily represent official NIH views.

\section{References}

1. National Hospital Discharge Survey (NHDS), National Center for Health Statistics. (2015). Accessed: February 1, 2019: https://www.cdc.gov/nchs/products/databriefs/db182.htm.

2. Beaudoin FL, Haran JP, Liebmann O: A comparison of ultrasound-guided three-in-one femoral nerve block versus parenteral opioids alone for analgesia in emergency department patients with hip fractures: a randomized controlled trial. Acad Emerg Med. 2013, 20:584-591. 10.1111/acem.12154

3. Morrison RS, Dickman E, Hwang U, et al.: Regional nerve blocks improve pain and functional outcomes in hip fracture: a randomized controlled trial. J Am Geriatr Soc. 2016, 64:2433-2439. 10.1111/ggs.14386

4. Sparks S, Evans D, Byars D: A low cost, high fidelity nerve block model. Crit Ultrasound J. 2014, 6:12. 10.1186/s13089-014-0012-2

5. Grossman KA, Fishman EK, Horton KM, Raman SP: Media leadership: change management. J Am Coll Radiol. 2017, 14:848-849. 10.1016/j.jacr.2017.01.014

6. Perkins MB, Jensen PS, Jaccard J, Gollwitzer P, Oettingen G, Pappadopulos E, Hoagwood KE: Applying theory-driven approaches to understanding and modifying clinicians' behavior: what do we know? Psychiatr Serv. 2007, 58:10.1176/ps.2007.58.3.342

7. Prosci change management methodology. (2010). Accessed: April 19, 2017: https://www.prosci.com/resources/articles/change-management-methodology.

8. Feletto E, Lui GW, Armour C, Saini B: Practice change in community pharmacy: using change-management principles when implementing a pharmacy asthma management service in NSW, Australia. Int J Pharm Pract. 2013, 21:28-37. 10.1111/j.2042-7174.2012.00225.x

9. Kotter JP, Kim WC, Mauborgne R: HBR's 10 Must Reads on Change Management . Harvard Business Review 


\section{Cureus}

Press (ed): Harvard Business School Publishing Corporation, Boston, MA; 2011. 10.1002/pmj.21413

10. Marrow J, Toney-Butler T: Change management. StatPearls [Internet]. 2019, Accessed: February 1, 2019: https://www.ncbi.nlm.nih.gov/books/NBK459380/. 